\title{
Study of Heterosis and Combining Ability for Yield and its Component Traits in Brassica juncea $\mathrm{L}$.
}

\author{
S.K. Rai ${ }^{1}$, Rubby Sandhu ${ }^{1}$, Lekhraj Jat ${ }^{1}$, Ashwani Kumar ${ }^{1}$, Usha Kiran ${ }^{2}$, \\ Shazia Mukhtar ${ }^{2}$, Surbhi Kohli ${ }^{1}$ and G.K. Rai ${ }^{2}$ \\ ${ }^{1}$ Division of Plant Breeding and Genetics, SKUAST-Jammu, Chatha, Jammu 18009 (J\&K), India \\ ${ }^{2}$ School of biotechnology SKUAST-Jammu, Chatha, Jammu 18009 (J\&K), India \\ *Corresponding author
}

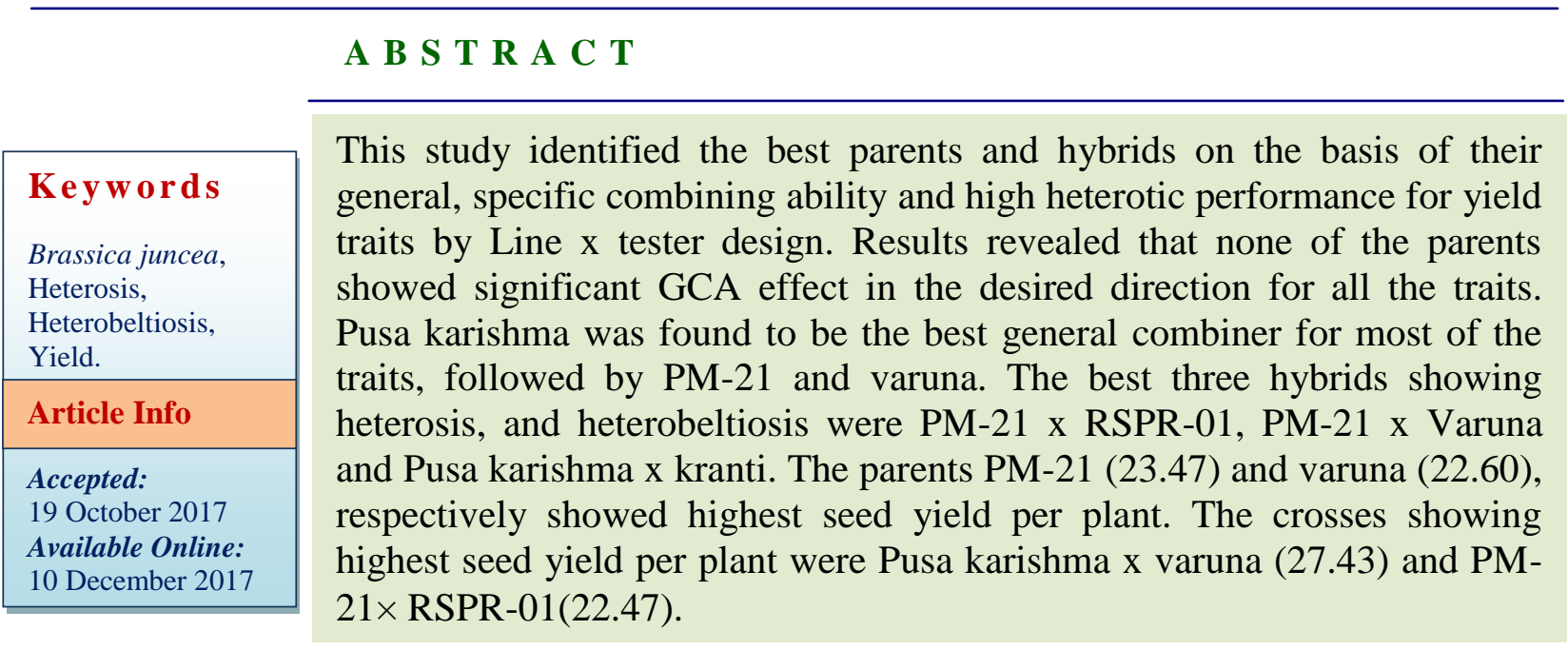

\section{Introduction}

In Indian subcontinent, $B$. juncea is the dominant species growing along with $B$. rapa (syn. B. campestris L.) and B. napus L. and these are the important sources of edible oil in India. Brassica juncea is commonly known as Indian mustard. It is an amphidiploid of $B$. rapa $(2 \mathrm{n}=20 ;$ genome $\mathrm{AA})$ and $B$. nigra ( $2 \mathrm{n}=16$; genome $\mathrm{BB}$ ) having 36 chromosomes and genome AAB Heterosis breeding approach is the most successful technological options being employed for the improvement of crop varieties. Heterosis is the interpretation of increased vigor, manifested by cross bred as compared with corresponding inbreds. Heterosis breeding could be used for enhancing crop productivity in Brassica juncea, a major oilseed crop of the Indian subcontinent. It is an important tool to overcome the existing yield barriers. For developing promising varieties through hybridization a careful choice of parents and breeding methodology are matters of great concern to a plant breeder.

Identifying parental material combination with strong heterosis for yield and obtain genetic parameters are the important steps in the development of new cultivars. It is important to have information about the desirable parental combinations which can 
represent a high degree of heterotic response. By exploiting heterosis in the $F_{1}$ hybrids, production cost could be reduced by increasing yield level and enhancing input use efficiency (Pingali, 1997).

Indian mustard being a self-pollinated crop, the technique of line $\mathrm{x}$ tester of Kempthorne (1957) for combing ability analysis is very important for screening lines with rapidity.

\section{Experimental material}

The experimental material for the present investigation consisted of ten diverse varieties / strains of Brassica juncea L. procured from the genetic stock available with oil seeds breeding section of Divison of Genetics and Plant Breeding, SKUAST-J, F.O.A Chatha.

Out of which six lines were used as females and four were used as tester (males). This parental material was grown in Randomized Complete Block Design with three replications in rabi season 2013- 2014. Genotypes along with their pedigree are shown in Table 1.

\section{Development of $F_{1}$ hybrids}

At the flowering, the six lines and four tester were crossed using Line $x$ Tester mating design through hand emasculations and controlled pollinations. Butter paper bags were used for avoiding contaminations. Twenty four $F_{1}$ were derived from these crosses and were grown in RCBD with three replications in rabi season 2014- 2015.

\section{Experimental observations}

Five plants were randomly selected from each treatment in each replication for recording the observations. These plants were tagged and detailed observations were recorded on the selected traits.

\section{Statistical and biometrical analysis}

Statistical analysis such as combining ability analysis (GCA and SCA) and Heterosis estimation was done using viva Statistical Analysis System (SAS) Software version 9.3.

\section{Analysis of variance}

The analysis of variance for the design of experiment was carried out according to the procedure outlined by Panse and Sukhatme (1967). The significance of difference among treatment means was tested by 'F' test.

The data collected from 34 genotypes (ten parents and twenty four $F_{1}$ 's) for yield and quality attributes were subjected to analysis of variance to determine the significant differences among various genotype means. The characters showing significant genotypic differences were further analyzed.

\section{The combining ability analysis}

\section{Analysis of variance for combining ability effects}

The ANOVA for general combining ability effects of the parents and specific combining ability effects of the crosses were worked out as suggested by Kempthorne (1957).

\section{Estimation of GCA and SCA effects}

GCA effect of lines $\left(\mathrm{g}_{\mathrm{i}}\right)=\frac{\frac{x i_{\mathrm{m}}}{r t}-\frac{x_{\mathrm{m}}}{r l t}}{r l}$
GCA effects of tester $\left(\mathrm{g}_{\mathrm{j}}\right)=\frac{\frac{x_{\mathrm{j}} j_{.}}{r l}}{r l}-\frac{x_{\mathrm{m}}}{r l t}$

SCA effects of hybrids $\left(\mathrm{s}_{\mathrm{ij}}\right)=$ $\frac{x i j_{0}}{r}-\frac{x i_{0}}{r t}-\frac{x_{. j} j_{0}}{r l}+\frac{x_{m}}{r l t}$

Where,

$\mathrm{x}_{\ldots}=$ Total of all hybrids over replications 
$\mathrm{x}_{\mathrm{i..}}=$ Total of ith line over $\mathrm{t}$ tester and $\mathrm{r}$ replications

$\mathrm{x} . \mathrm{j} .=$ Total of $\mathrm{jth}$ tester over 1 line and $\mathrm{r}$ replications

xij. $=$ Total of the hybrids between ith line and $\mathrm{jth}$ tester over $\mathrm{r}$ replications

\section{Standard error for combining ability effects}

SE for GCA effects of lines $=\sqrt{\frac{\text { EMS }}{r t}}$

SE for GCA effects of tester $=\sqrt{\frac{E M S}{r l}}$

SE for SCA effects of hybrids $=\frac{\sqrt{E M S}}{r}$

Test of significance of GCA and SCA effects by ' $t$ ' test

$t=\frac{E f f e c t}{S E}$

\section{Estimation of heterosis}

Heterosis is expressed as percent increase or decrease in the performance of $F_{1}$ hybrid over the mid parent (relative or mid parent heterosis) and better parent (heterobeltiosis) was computed for each character using standard formulas (Shull, 1952).

\section{Relative Heterosis}

The superiority of $F_{1}$ hybrid over the midparental value (i.e., mean value of two parents involved in the cross is known as mid-parent or relative heterosis.

Relative Heterosis $=\frac{F 1-M P}{M P} \times 100$

\section{Heterobeltiosis}

The superiority of $F_{1}$ hybrid over the betterparent out of the two parents involved in the cross is referred to as better-parent heterosis or heterobeltiosis.

Heterobeltiosis $=\frac{F 1-B P}{B P} \times 100$

Where,

$F_{1}=$ Mean performance of $F_{1}$ hybrid

$\mathrm{P}_{1}=$ Mean performance of parent one

$\mathrm{P}_{2}=$ Mean performance of parent two

$M P=$ Mean performance of mid parent

$B P=$ Mean performance of better parent

Standard error for relative Heterosis = $\sqrt{\frac{3}{2 r}}$ EMS

Standard error for Heterobeltiosis $=\sqrt{\frac{2}{r}}$ EMS

Where, EMS = Error mean square from ANOVA table and $r=$ number of replications

\section{Test of significance}

The significance of heterosis was tested at error degrees of freedom by the formula as suggested by Turner (1953).

' $\mathrm{t}$ ' value for mid-parent Heterosis $=\underset{\frac{F 1-B P}{S E}}{S E}$
' $\mathrm{t}$ ' value for heterobeltiosis $=\frac{F 1-M P}{S E}$
Where, $\mathrm{SE}=$ standard error

\section{Analysis of Variance}

\section{Analysis of variance for line $\mathrm{x}$ tester design}

The analysis of variance revealed considerable genetic variation among parents and hybrids for almost all the traits under study (Table 2).

The mean sum of squares for parents was highly significant for all the characters which indicated the presence of sufficient variability 
among parents. Highly significant mean square variances for almost all the characters were also observed in case of lines and tester, which indicated the presence of additive variance. Highly significant mean sum of squares for line $\times$ tester for plant height $(\mathrm{cm})$, siliqua length $(\mathrm{cm})$, number of of seeds per siliqua, 1000-seed weight (g), seed yield per plant $(\mathrm{g})$, harvest index $(\%)$ indicated the presence of dominance variance.

The mean sum of squares for hybrids was also highly significant, which indicated the diverse performance of different cross combinations for all traits except for siliqua lengh $(\mathrm{cm})$. The parents versus hybrids mean sum of squares were highly significant for most of the traits, which revealed the presence of heterosis due to the significant difference in the mean performance of hybrids and parents.

\section{Estimates of heterosis}

The extent of heterosis was estimated as per cent increase or decrease of $\mathrm{F}_{1}$ hybrid over mid parent (Table 3) and better parent (Table 4). The results have been described character wise here under:

\section{Plant height (cm)}

Manifestation of heterosis was found in both positive and negative directions. The mid parent heterosis ranged from out of twenty four crosses, four hybrids viz., PM-22 x RSPR-03 (-23.85**), PM-24 x RSPR-03 (20.42**), PM-22 x RSPR-01 (-17.43**) showed negative mid parent heterosis which is advantageous to develop short statured genotypes in Brassica to avoid lodging.

Whereas as only two hybrids viz. PM-24 $\mathrm{x}$ Kranti (11.31**), Nov gold x Kranti (10.8**) showed highly significant positive heterosis. Ten crosses showed negative heterobeltiosis for plant height.

\section{Number of primary branches per plant}

The estimates for heterosis revealed that nine out of twenty four hybrids viz., PM-24 $\mathrm{x}$ RSPR-01 (50**), Pusa karishma $\mathrm{x}$ varuna (28.57**), Pusa karishma X RSPR-03 (26.83**), Pusa karishma $\mathrm{x}$ RSPR-01 (24.32**), PM-24 x RSPR-03 (11.11**), PM$22 \mathrm{x}$ Varuna $(7.32 * *)$, Pusa karishma x kranti (7.32**), PM-22 x RSPR-01 (5.56**) exhibited highly significant positive heterosis number of primary branches per plant. Eight crosses showed positive heterobeltiosis for number of primary branches per plant. PM-24 $\mathrm{x}$ RSPR-01 (50.00**) exhibited highest heterobeltiosis.

\section{Number of secondary branches per plant}

The estimates for heterosis revealed that nine hybrids viz., Pusa karishma x RSPR-03 (86.11**), Pusa karishma x kranti (70.73**), PM-24 x RSPR-01 (52.24**), PM-21x RSPR03 (35**), PM-22 x RSPR-01 (27.27**), PM24 x RSPR-03 (26.98**), Pusa karishma $\mathrm{x}$ RSPR-01 (21.05**), PM-22 x RSPR-03 (19.35**), Nov gold x RSPR-03 (7.25**) exhibited highly significant positive heterosis number of secondary branches per plant. Nine crosses showed positive heterobeltiosis for number of secondary branches per plant (Table 6). The top three crosses showing highest heterobeltiosis were Pusa karishma $\mathrm{x}$ kranti (66.67**), Pusa karishma x RSPR-03 (59.52**) and PM-24 x RSPR-01 (50.00**), respectively.

\section{Number of siliquae on main raceme}

The results for heterosis estimates for number of siliquae on main raceme revealed that highly significant positive mid-parental heterosis was displayed by five crosses viz., Pusa karishma $\mathrm{x}$ kranti $(23.53 * *)$, Pusa karishma x RSPR-03 (9.65**), NRCDR-2 x kranti $\left(7.46^{* *}\right)$, Nov gold x Kranti $(6.74 * *)$. 
Only two crosses viz., Pusa karishma x kranti (9.57**) and Nov gold x Kranti (6.74**) showed positive heterobeltiosis.

\section{Siliqua length $(\mathrm{cm})$}

Twelve out twenty-four hybrids displayed significant positive mid-parental heterosis for siliqua length viz., Nov gold x RSPR-01 (25.58**), Pusa karishma x kranti (20.71**), Nov gold $\mathrm{x}$ Kranti (15.79**), Nov gold $\mathrm{x}$ Varuna (11.83**), PM-22 x Varuna (7.39**), Nov gold $\mathrm{x}$ RSPR-03 (7.37**), PM-22 x RSPR-01 (6.88**), PM-24 x RSPR-01 (6.88**), PM-22 x Kranti (6.38**), Pusa karishma x RSPR-01 (5.88*), NRCDR-2 x varuna $\left(5.71^{*}\right), \mathrm{PM}-21 \times \operatorname{Varuna}\left(5.53^{*}\right)$. Seven crosses showed positive heterobeltiosis for siliqua length.

\section{Number of seeds per siliqua}

The results for heterosis estimates (Table 5) for number of seeds per siliqua showed that highly significant positive mid-parental heterosis was displayed by six crosses viz., Pusa karishma x RSPR-01 (37.5**) Pusa karishma x kranti (25.71**), PM-22 x RSPR01 (18.84**), Pusa karishma x RSPR-03 (17.14**), PM-24 x RSPR-01 (8.11**), PM22 x RSPR-03 (6.67**). Four crosses showed positive heterobeltiosis.

\section{0-seed weight (g)}

The estimates of heterosis (Table 5) for 1000seed weight revealed that all the crosses exhibited highly significant mid-parental heterosis but none of the hybrids showed heterosis in desired direction. Only a single cross viz., PM-22 x Kranti (10.99**) showed positive heterobeltiosis.

\section{Seed yield per plant (g)}

The estimates of heterosis for seed yield revealed that highly significant positive mid- parental heterosis was displayed by eight crosses viz., PM-24 x RSPR-01 (32.15**), Pusa karishma $\mathrm{x}$ varuna $(30.32 * *)$, Pusa karishma x RSPR-01 (27.35**), PM-22 x RSPR-01 (25.83**), Nov gold x RSPR-03 (16.03**), PM-22 x RSPR-03 (12.43**) Nov gold x RSPR-01 (7.62**), PM-24 x Varuna $(2.09 * *)$. Five crosses viz., Pusa karishma $\mathrm{x}$ varuna (21.57**), PM-22 x RSPR-01 (20.00**), PM-24 x RSPR-01 (18.26**), PM22 x RSPR-03 (7.32**) and Pusa karishma $\mathrm{x}$ RSPR-01 (4.09**), showed highly significant heterobeltiosis for seed yield

\section{Harvest index (\%)}

Five hybrids viz., Nov gold x RSPR-01 (8.76**), PM-24 x RSPR-01 (2.44*), Nov gold x RSPR-01 (2.37**), PM-21× Kranti (2.20**), PM-24 x RSPR-03 (1.35**) exhibited positively significant mid-parental heterosis for harvest index (Table 5). Only one cross viz., Nov gold x RSPR-01 (8.11**) showed positive heterobeltiosis (Table 6).

\section{Line X tester analysis of variance}

The line $\times$ tester analysis have been used by several Brassica breeders for the genetic analysis of morphological traits, estimation of GCA and SCA, evaluation of heterosis in Brassicas and other oilseed crops (Dar et al., 2013). Results of ANOVA for line $x$ tester design revealed highly significant values for parents, crosses, and parents vs. crosses indicating sufficient genetic variability in the material for all the characters under study. Comparison of mean squares due to parents vs. hybrids were found highly significant for plant height, number of siliqua on main raceme, seed yield per plant, harvest index, and all the characters under study except number of primary branches/ plant, siliqua length, number of seeds per siliqua, 1000seed weight and number of secondary branches per plant, indicating that mean of hybrids were significantly differ from that of 
the parents as a group for these traits by suggesting the presence of mean heterosis for all these characters. Similar result was reported by Patel et al., (2015), Yadava et al., (2012).The analysis of variance for combining ability revealed that the mean squares due to lines were significant for all the traits except 1000-seed weight. This indicated significant contribution of lines towards gca variance component for these traits. Similar result was reported by Patel et al., (2015). The mean square due to lines were greater than those due to tester for almost all the traits except plant height, which is indicating large diversity among the lines than in tester for these characters. Similar result was reported by Patel et al., (2015). The line $\mathrm{x}$ tester interaction was significant for all the characters except siliqua length and 1000seed weight. Indicating non additive genetic effects have important role for controlling these traits.

Table.1 Analysis of variance for Lines x Testers design

\begin{tabular}{|c|c|c|c|}
\hline Sources & d.f. & MSS & EMS \\
\hline Replication (r) & $(r-1)$ & & \\
\hline Genotypes $(g)$ & $(g-1)$ & $\mathrm{MS}_{2}$ & \\
\hline Parents $(\mathrm{p})$ & $(\mathrm{p}-1)$ & & \\
\hline Crosses $(\mathrm{c})$ & $(\mathrm{c}-1)$ & & \\
\hline Parents vs Crosses & 1 & & \\
\hline Lines (1) & $(1-1)$ & $\mathrm{M}_{1}$ & $\sigma_{e}^{2}+\mathrm{r}(\operatorname{Cov} . \mathrm{F} . \mathrm{S}-2 . \mathrm{Cov} . \mathrm{H} . \mathrm{S})+\mathrm{rt}(\mathrm{Cov} . \mathrm{H} . \mathrm{S})$ \\
\hline Testers $(\mathrm{t})$ & $(\mathrm{t}-1)$ & $\mathrm{M}_{\mathrm{t}}$ & $\sigma_{e}^{2}+\mathrm{r}($ Cov.F.S - 2.Cov.H.S $)+\mathrm{rl}$ (Cov.H.S) \\
\hline Lines vs Testers & 1 & $\mathrm{M}_{\mathrm{l}} \times \mathrm{M}_{\mathrm{t}}$ & $\sigma_{e}^{2}+\mathrm{r}($ Cov.F.S $-2 . \operatorname{cov} . \mathrm{H} . \mathrm{S})$ \\
\hline Error & $(r-1)(\mathrm{g}-1)$ & $\mathrm{MS}_{1}$ & $\sigma_{a}^{2}$ \\
\hline Total & Ltr-1 & & \\
\hline
\end{tabular}

Where $\mathrm{MS}_{2}, \mathrm{M}_{1}, \mathrm{M}_{t}, \mathrm{M}_{1} \mathrm{xM}_{\mathrm{t}}$, and $\mathrm{MS}_{1}$ were genotypic mean square, line mean square, tester mean square, line vs tester mean square, and error mean square, respectively.

Table.2 ANOVA for combining ability

\begin{tabular}{|l|l|l|l|}
\hline Source of variation & d.f. & M.S. & Expected M.S. \\
\hline Replications & $(\mathrm{r}-1)$ & & \\
\hline Lines & $(\mathrm{l}-1)$ & $\mathrm{M}_{\mathrm{l}}$ & $\sigma^{2} \mathrm{e}+\mathrm{r}$ [Cov. (F.S) - 2 Cov. (H.S.)] + fr Cov. (H.S.) \\
\hline Testers & $(\mathrm{t}-1)$ & $\mathrm{M}_{\mathrm{t}}$ & $\sigma^{2} \mathrm{e}+\mathrm{r}$ [Cov. (F.S) -2 Cov. (H.S.) + mr Cov. (H.S.) \\
\hline Lines x Testers & $(\mathrm{l}-1)(\mathrm{t}-1)$ & $\mathrm{M}_{\mathrm{lxt}}$ & $\sigma^{2} \mathrm{e}+\mathrm{r}$ [Cov. (F.S) - 2 Cov. (H.S.)] \\
\hline Error & $(\mathrm{lt}-1)(\mathrm{r}-1)$ & $\mathrm{M}_{\mathrm{e}}$ & $\sigma^{2} \mathrm{e}$ \\
\hline Total & $(\mathrm{ltr}-1)$ & & \\
\hline
\end{tabular}

Where $\mathrm{M}_{\mathrm{l}}, \mathrm{M}_{t}, \mathrm{M}_{\mathrm{lxt}}$, and $\mathrm{M}_{\mathrm{e}}$ were line mean square, tester mean square, line $\mathrm{x}$ tester mean square, and error mean square, respectively.

Table.3 Genotypes along with their pedigree

\begin{tabular}{|c|c|c|c|}
\hline S. No. & Genotypes & Species & Pedigree \\
\hline \multicolumn{4}{|c|}{ Lines } \\
\hline 1. & PM-21 & Brassica juncea & Pusa bold x Zem-2 \\
\hline 2. & PM-22 & Brassica juncea & Pusa Barani x Zem-2 \\
\hline 3. & PM-24 & Brassica juncea & (Pusa Bold x LEB-15) x LES-29 \\
\hline 4. & Pusa Karishma & Brassica juncea & Pusa Basanti x Zem-1 \\
\hline 5. & Nov-Gold & Brassica juncea & Bio-902×BM-185-11 \\
\hline 6. & NRCDR-2 & Brassica juncea & MDOC-43×NBPGR-36 \\
\hline \multicolumn{4}{|c|}{ Tester } \\
\hline 7. & RSPR-01 & Brassica juncea & B.juncea $\times$ D.muralis \\
\hline 8. & RSPR-03 & Brassica juncea & Kranti×Pusa Bold \\
\hline 9. & Varuna & Brassica juncea & Selection from Varanasi Local 786,02.021976 \\
\hline 10. & Kranti & Brassica juncea & Selection from Varuna \\
\hline
\end{tabular}


Table.4 Analysis of variance for Lines x Testers for seed yield and yield contributing traits in Brassica juncea

\begin{tabular}{|c|c|c|c|c|c|c|c|c|c|c|}
\hline $\begin{array}{l}\text { Sources / } \\
\text { Characters }\end{array}$ & d.f & $\begin{array}{l}\text { Plant height } \\
(\mathrm{cm})\end{array}$ & $\begin{array}{c}\text { No. of } \\
\text { primary } \\
\text { branches/ } \\
\text { plant }\end{array}$ & $\begin{array}{c}\text { No. of } \\
\text { secondary } \\
\text { branches/ } \\
\text { plant }\end{array}$ & $\begin{array}{l}\text { No. of siliqua } \\
\text { on main } \\
\text { raceme }\end{array}$ & $\begin{array}{l}\text { Siliqua } \\
\text { length } \\
(\mathrm{cm})\end{array}$ & $\begin{array}{l}\text { No. of } \\
\text { seeds } \\
\text { /siliqua }\end{array}$ & $\begin{array}{l}\text { 1000-seed } \\
\text { weight }(\mathrm{g})\end{array}$ & $\begin{array}{l}\text { Seed yield } \\
\text { per } \\
\text { plant }(\mathrm{g})\end{array}$ & $\begin{array}{c}\text { Harvest } \\
\text { index }(\%)\end{array}$ \\
\hline Replication & 2 & 25.80 & 1.24 & 0.42 & 3.30 & 0.13 & 0.07 & $0.02 *$ & 2.87 & 1.22 \\
\hline Parents & 9 & $1493.57 * *$ & $1.88^{*}$ & $25.50 * *$ & $168.74 * *$ & $0.42 * *$ & $9.72 * *$ & $0.878^{* *}$ & $57.47 * *$ & $15.67 * *$ \\
\hline Lines & 5 & $190.23 * *$ & $2.09 *$ & $24.90 * *$ & $197.26^{* *}$ & $0.36^{* *}$ & $10.36^{* *}$ & $0.69 * *$ & $54.60 * *$ & $9.30 * *$ \\
\hline Testers & 3 & $1462.03 * *$ & 1.64 & $33.64 * *$ & $177.11 * *$ & $0.31 *$ & 5.67 & $1.47 * *$ & $72.49 * *$ & $3.74 * *$ \\
\hline Lines vs Testers & 1 & $3676.30 * *$ & 1.61 & 4.05 & 1.09 & $1.10^{* *}$ & $18.69 * *$ & $0.078^{* *}$ & $26.76^{* * *}$ & $78.87 * *$ \\
\hline Crosses & 23 & $577.08 * *$ & $4.35 * *$ & $36.25 * *$ & $111.76^{* *}$ & 0.17 & $8.13 * *$ & $0.34 * *$ & $27.76^{* * *}$ & $5.94 * *$ \\
\hline $\begin{array}{c}\text { Parents vs } \\
\text { Crosses }\end{array}$ & 1 & $1048.44^{* *}$ & 0.22 & $14.03 *$ & $706.24 * *$ & 0.078 & 6.34 & $13.98 * *$ & $35.70^{* *}$ & $51.42 * *$ \\
\hline Error & 66 & 23.07 & 0.799 & 3.09 & 9.78 & 0.11 & 2.25 & 0.01 & 1.23 & 0.47 \\
\hline
\end{tabular}

$*$, ** significant at 0.05 and 0.01 respectively

Table.5 Estimates of relative heterosis of crosses for seed yield and yield contributing traits in Brassica juncea

\begin{tabular}{|c|c|c|c|c|c|c|c|c|c|}
\hline Hybrids & $\mathrm{PH}$ & PB & SB & SMR & SL & SS & TW & $\mathrm{HI}$ & SY \\
\hline PM-21 × Varuna & -2.69 & $-25.58 * *$ & $-35.92 * *$ & $-9.96 * *$ & $5.53 *$ & $-13.33 * *$ & $-19.13 * *$ & $-30.85 * *$ & $-23.97 * *$ \\
\hline PM-21× Kranti & 1.11 & $-38.18 * *$ & $-31.11 * *$ & $-31.62 * *$ & -2.17 & $-11.64 * *$ & $-6.31 * *$ & $-10.22 * *$ & $-17.09 * *$ \\
\hline PM-21 x RSPR-01 & -4.09 & $15.79 * *$ & $42.86 * *$ & $-55.56 * *$ & $8.11 * *$ & $5 * *$ & $-3.98 * *$ & $2.20 * *$ & $25.28 * *$ \\
\hline PM-21x RSPR-03 & -1.42 & $19.05 * *$ & $35 * *$ & $-57.79 * *$ & $-9.36 * *$ & $-6.98 * *$ & $-28.35 * *$ & $-11.41 * *$ & $-9.09 * *$ \\
\hline PM-22 x Varuna & -2.67 & $7.32 * *$ & 1.18 & $-9.92 * *$ & $7.39 * *$ & $-11.39 * *$ & $-31.97 * *$ & $-21.20 * *$ & $-9.11 * *$ \\
\hline PM-22 x Kranti & -0.66 & $-20 * *$ & $-5.56 * *$ & -2.44 & $6.38 * *$ & $-17.33 * *$ & $-5.71 * *$ & $-16.91 * *$ & $-12.74 * *$ \\
\hline PM-22 x RSPR-01 & $\begin{array}{c}- \\
17.43^{* *}\end{array}$ & $5.56 * *$ & $27.27 * *$ & $-17.84 * *$ & $6.88 * *$ & $18.84 * *$ & $-15.15 * *$ & $-8.57 * *$ & $25.83 * *$ \\
\hline PM-22 x RSPR-03 & $\begin{array}{c}- \\
23.85^{* *} \\
\end{array}$ & $-20 * *$ & $19.35 * *$ & $-23.85 * *$ & $\begin{array}{c}- \\
21.74 * * \\
\end{array}$ & $6.67 * *$ & $-23.28 * *$ & $-9.02 * *$ & $12.43 * *$ \\
\hline PM-24 x Varuna & -1.34 & $18.92 * *$ & 0 & $-27.66 * *$ & 0.49 & $-7.14 * *$ & $-18.17 * *$ & $-8.25 * *$ & $2.09 * *$ \\
\hline PM-24 x Kranti & $11.31 * *$ & $-5.56 * *$ & $-6.85^{* *}$ & $-18.37 * *$ & -1.06 & $-20 * *$ & $-14.25 * *$ & $-14.40 * *$ & $-15.54 * *$ \\
\hline PM-24 x RSPR-01 & 0 & $50 * *$ & $52.24 * *$ & $-12.46^{* *}$ & $6.88 * *$ & $8.11 * *$ & $-9.77 * *$ & $2.44 * *$ & $32.15^{* *}$ \\
\hline PM-24 x RSPR-03 & $\begin{array}{c}- \\
20.42 * *\end{array}$ & $11.11 * *$ & $26.98 * *$ & $-31.33 * *$ & $\begin{array}{c}- \\
13.04 * *\end{array}$ & $-5 * *$ & $-26.66 * *$ & $1.35^{* *}$ & $-5.42 * *$ \\
\hline $\begin{array}{c}\text { Pusa karishma x } \\
\text { varuna }\end{array}$ & -6.76 & $28.57 * *$ & $-7.37 * *$ & 2.9 & 4.35 & $2.7 *$ & $-27.97 * *$ & $2.37 * *$ & $30.32 * *$ \\
\hline Pusa karishma x kranti & $6.8 *$ & $7.32 * *$ & $70.73 * *$ & $23.53 * *$ & $20.71 * *$ & $25.71 * *$ & $-12.29 * *$ & $-17.2 * *$ & $-13.52 * *$ \\
\hline $\begin{array}{c}\text { Pusa karishma } \mathrm{x} \\
\text { RSPR-01 }\end{array}$ & -1.96 & $24.32 * *$ & $21.05 * *$ & $-11.67 * *$ & $5.88 *$ & $37.5 * *$ & $-23.63 * *$ & $-5.29 * *$ & $27.35^{* *}$ \\
\hline $\begin{array}{c}\text { Pusa karishma x } \\
\text { RSPR-03 }\end{array}$ & 1.83 & $26.83 * *$ & $86.11 * *$ & $9.65 * *$ & $-4.26^{*}$ & $17.14 * *$ & $-29.88 * *$ & $-11.86 * *$ & $-6.65^{* *}$ \\
\hline Nov gold $\mathrm{x}$ Varuna & -3.45 & $-27.27 * *$ & $-15.22 * *$ & -1.4 & $11.83 * *$ & $-30.12 * *$ & $-18.63 * *$ & $-17.12 * *$ & $-27.97 * *$ \\
\hline Nov gold x Kranti & $10.8 * *$ & $-16.28 * *$ & $-18.99 * *$ & $6.74 * *$ & $15.79 * *$ & $-34.18 * *$ & $-4.89 * *$ & $-15.02 * *$ & $-35 * *$ \\
\hline Nov gold x RSPR-01 & -0.38 & $-7.69 * *$ & $4.11 * *$ & 0.93 & $25.58 * *$ & $-15.07 * *$ & $-15.55 * *$ & $8.76 * *$ & $7.62 * *$ \\
\hline Nov gold x RSPR-03 & -2.13 & $-11.63 * *$ & $7.25 * *$ & $-8.15 * *$ & $7.37 * *$ & $-11.39 * *$ & $-20.97 * *$ & $-5.73 * *$ & $16.03 * *$ \\
\hline NRCDR-2 $x$ varuna & -6.4 & $-20.93 * *$ & $-20.75 * *$ & $-6.72 * *$ & $5.71 *$ & $-18.6^{* *}$ & $-36.75^{* *}$ & $-15.63 * *$ & $-29.39 * *$ \\
\hline NRCDR-2 x kranti & 4.54 & $-19.05 * *$ & $-20.43 * *$ & $7.46 * *$ & -1.54 & $-26.83 *$ & $-7.82 * *$ & $-21.35 * *$ & $-35.11 * *$ \\
\hline NRCDR-2 x RSPR-01 & -3.62 & $5.26 * *$ & $-10.34 * *$ & $-13.08 * *$ & $5.1 *$ & $-2.63 *$ & $-13.98 * *$ & $-8.10 * *$ & $-20.96 * *$ \\
\hline NRCDR-2 x RSPR-03 & -2.72 & $-4.76 * *$ & $-3.61 * *$ & $-13.28 * *$ & -4.67 & 2.44 & $-24.91 * *$ & $-1.83 * *$ & $-5.68 * *$ \\
\hline S.E. & 3.396 & 0.629 & 1.243 & 2.221 & 2.294 & 1.061 & 0.044 & 0.56 & 0.785 \\
\hline
\end{tabular}

$*$, ** significant at 0.05 and 0.01 respectively. Abbreviations used: $\mathrm{PH}=\mathrm{Plant}$ height, $\mathrm{PB}=$ Number of primary

branches, $\mathrm{SB}=$ Number of secondary branches, $\mathrm{SMR}=$ Number of siliquae on main raceme $\mathrm{SP}=\mathrm{Number}$ of siliquae

per plant $\mathrm{SS}=$ Number of seeds per siliqua, $\mathrm{TW}=1000$ seed weight, $\mathrm{SY}=$ seed yield per plant, $\mathrm{HI}=\mathrm{Harvest} \mathrm{Index}$ 
Table.6 Estimates of Heterobeltiosis of crosses for seed yield and yield contributing traits in Brassica juncea

\begin{tabular}{|c|c|c|c|c|c|c|c|c|c|}
\hline crosses/ characters & Plant height $(\mathrm{cm})$ & $\begin{array}{c}\text { No. of } \\
\text { primary } \\
\text { branches/ } \\
\text { plant }\end{array}$ & $\begin{array}{c}\text { No. of } \\
\text { secondary } \\
\text { branches/ } \\
\text { plant }\end{array}$ & $\begin{array}{c}\text { No. of } \\
\text { siliqua on } \\
\text { main } \\
\text { raceme }\end{array}$ & $\begin{array}{l}\text { Siliqua } \\
\text { length } \\
(\mathrm{cm})\end{array}$ & $\begin{array}{l}\text { No. of seeds } \\
\text { /siliqua }\end{array}$ & $\begin{array}{c}\text { 1000-seed } \\
\text { weight } \\
\text { (g) }\end{array}$ & $\begin{array}{l}\text { Seed yield per } \\
\text { plant }(\mathrm{g})\end{array}$ & $\begin{array}{c}\text { Harvest } \\
\text { index }(\%)\end{array}$ \\
\hline PM-21 $\times$ Varuna & -3.38 & $-27.27 * *$ & $-37.74 * *$ & $-15.86^{* *}$ & $1.94 * *$ & $-15.22 * *$ & $-24.86 * *$ & $-25.43 * *$ & $-36.84 * *$ \\
\hline PM-21× Kranti & $-17.51 * *$ & $-40.91 * *$ & $-38.00 * *$ & $-44.83 * *$ & $-12.62 * *$ & $-13.64 * *$ & $-15.51 * *$ & $-20.74 * *$ & $-13.11 * *$ \\
\hline PM-21 x RSPR-01 & -4.87 & 0.00 & $20.00 * *$ & $-58.62 * *$ & $-2.91 * *$ & $-4.55 * *$ & $-5.37 * *$ & $-4.26 * *$ & $-5.47 * *$ \\
\hline PM-21x RSPR-03 & -2.81 & $13.64 * *$ & $8.00 * *$ & $-57.93 * *$ & $-10.68 * *$ & $-9.09 * *$ & $-33.73 * *$ & $-25.43 * *$ & $-19.65 * *$ \\
\hline PM-22 x Varuna & -2.67 & $4.76 * *$ & $-18.87 * *$ & $-13.49 * *$ & $1.87 * *$ & $-23.91 * *$ & $-34.21 * *$ & $-27.03 * *$ & $-34.89 * *$ \\
\hline PM-22 x Kranti & $-19.40 * *$ & $-20.00 * *$ & $-15.00 * *$ & $-13.79 * *$ & $-6.54 * *$ & $-26.19 * *$ & $10.99 * *$ & $-28.50 * *$ & $-27.87 * *$ \\
\hline PM-22 x RSPR-01 & $-18.68 * *$ & $-5.00 * *$ & $23.53^{* * *}$ & $-20.80 * *$ & $-5.61 * *$ & $13.89 * *$ & $-12.74 * *$ & $20.00^{* * *}$ & $-9.75 * *$ \\
\hline PM-22 x RSPR-03 & $-24.39 * *$ & $-20.00 * *$ & $15.62 * *$ & $-31.25 * *$ & $-24.30 * *$ & $-4.76 * *$ & $-26.16^{* *}$ & $7.32 * *$ & $-9.71 * *$ \\
\hline PM-24 x Varuna & -1.42 & $4.76 * *$ & $-18.87 * *$ & $-34.62 * *$ & $-4.67 * *$ & $-15.22 * *$ & $-23.44 * *$ & $-13.44 * *$ & $-27.20 * *$ \\
\hline PM-24 x Kranti & $-9.63 *$ & $-15.00 * *$ & $-15.00 * *$ & $-35.90 * *$ & $-13.08 * *$ & $-23.81 * *$ & $-2.98 * *$ & $-26.79 * *$ & $-32.27 * *$ \\
\hline PM-24 x RSPR-01 & -1.43 & $50.00 * *$ & $50.00^{* * *}$ & $-21.15 * *$ & $-5.61 * *$ & $5.26 * *$ & $-9.11 * *$ & $18.26^{* *}$ & $-2.68 * *$ \\
\hline PM-24 x RSPR-03 & $-21.05^{* *}$ & 0.00 & $21.21 * *$ & $-33.97 * *$ & $-15.89 * *$ & $-9.52 * *$ & $-31.70 * *$ & $-7.43 * *$ & $-4.28 * *$ \\
\hline $\begin{array}{c}\text { Pusa karishma } \mathrm{x} \\
\text { varuna }\end{array}$ & $-7.97 *$ & $28.57 * *$ & $-16.98 * *$ & -1.59 & 0.00 & $-17.39 * *$ & $-35.91 * *$ & $21.57 * *$ & $-1.47 * *$ \\
\hline Pusa karishma x kranti & $-14.21 * *$ & $4.76 * *$ & 66.67 ** & $9.57 * *$ & $15.91 * *$ & $4.76^{* * *}$ & $-17.35^{* *}$ & $-17.29 * *$ & $-19.55^{* *}$ \\
\hline $\begin{array}{c}\text { Pusa karishma x } \\
\text { RSPR-01 }\end{array}$ & -4.68 & $9.52 * *$ & $9.52 * *$ & $-15.20 * *$ & $2.27 * *$ & $22.22 * *$ & $-28.13 * *$ & $4.09 * *$ & $-14.20 * *$ \\
\hline $\begin{array}{c}\text { Pusa karishma x } \\
\text { RSPR-03 }\end{array}$ & 1.21 & $23.81 * *$ & $59.52 * *$ & -1.39 & $-10.00 * *$ & -2.38 & $-37.87 * *$ & $-17.41^{* *}$ & $-20.83 * *$ \\
\hline Nov gold x Varuna & -7.83 & $-30.43 * *$ & $-26.42 * *$ & $-15.87 * *$ & $8.33 * *$ & $-36.96^{* *}$ & $-19.95 * *$ & $-28.02 * *$ & $-32.43 * *$ \\
\hline Nov gold x Kranti & -6.65 & $-21.74 * *$ & $-20.00 * *$ & $6.74 *$ & $10.00 * *$ & $-38.10 * *$ & $-20.70 * *$ & $-36.73 * *$ & $-27.84 * *$ \\
\hline Nov gold x RSPR-01 & -3.49 & $-21.74 * *$ & -2.56 & $-13.60 * *$ & $20.00 * *$ & $-16.22^{* *}$ & $-21.66 * *$ & $-16.67 * *$ & $8.11 * *$ \\
\hline Nov gold x RSPR-03 & -7.19 & $-17.39 * *$ & $-5.13^{* * *}$ & $-25.69 * *$ & $2.00 * *$ & $-16.67 * *$ & $-21.86^{* *}$ & $-3.39 * *$ & $-6.96^{* *}$ \\
\hline NRCDR-2 $x$ varuna & $-8.90 * *$ & $-22.73 * *$ & $-20.75^{* *}$ & $-11.90 * *$ & $-2.63 * *$ & $-23.91 * *$ & $-37.71 * *$ & $-31.08 * *$ & $-27.41 * *$ \\
\hline NRCDR-2 x Kranti & $-13.35^{* * *}$ & $-22.73 * *$ & $-30.19 * *$ & -3.57 & $-15.79 * *$ & $-28.57 * *$ & $-23.08 * *$ & $-38.26 * *$ & $-31.37 * *$ \\
\hline NRCDR-2 x RSPR-01 & -4.77 & $-9.09 * *$ & $-26.42 * *$ & $-17.60 * *$ & $-9.65 * *$ & $-7.50 * *$ & $-20.12 * *$ & $-39.80 * *$ & $-10.49 * *$ \\
\hline NRCDR-2 x RSPR-03 & -5.96 & $-9.09 * *$ & $-24.53 * *$ & $-22.92 * *$ & $-10.53 * *$ & 0.00 & $-25.69 * *$ & $-22.93 * *$ & $-3.62 * *$ \\
\hline S.E. & 3.921 & 0.726 & 1.435 & 2.553 & 0.265 & 1.225 & 0.05 & 0.906 & 0.56 \\
\hline
\end{tabular}

The reliability of such information depends much on diversity of material under study. The results of analysis of variance revealed that significant variability existed among the parents in regard to all the characters under investigation. Such divergence of parents leads to development of F1s that differed significantly among themselves for all characters.

The data for seed yield per plant revealed that nine and five crosses manifested significant positive relative heterosis and heterobeltiosis, respectively. The crosses that exhibited highly significant positive heterobeltiosis for seed yield were Pusa karishma x varuna $(21.57 * *)$, PM-22 x RSPR-01 (20.00**), PM-22 x RSPR-03 (18.26**), PM-22 x RSPR-03 (7.32**) and Pusa karishma $x$ RSPR-01 $(4.09 * *)$. Moderate level of heterosis for seed yield/plant, was also reported by Aher et al., (2009). Short and medium plant stature less vulnerable to lodging due to heavy winds is also preferred in Brassica. Negative heterosis, therefore, is useful regarding plant height. Similarly, shorter plants with greater numbers of branches are desirable due to their ability to withstand winds. In the present study, negative mid-parent and better heterotic values for plant height were noted for three and eight crosses, respectively. Pourdad and Sachan (2003) also reported significant negative heterosis for plant height in Brassica napus. Similarly, Nassimi et al., (2006) also obtained significant negative better-parent heterosis for plant height.

In Brassica, positive heterosis for number of primary branches is desirable, because plants with vigorous stature containing more 
branches provide opportunity for higher yields. Significant positive heterosis for number of primary branches was earlier reported by Turi et al., (2006) and Nasrin et al., (2011). Similarly, for number of siliqua on main raceme, the significant positive better parent heterosis was observed for two crosses viz., Pusa karishma $\mathrm{x}$ kranti and Nov gold $\mathrm{x}$ Kranti with the values from 9.57 and 6.74 (\%), respectively. Eight crosses for siliqua length, four crosses for number of seeds per siliqua, and one cross for 1000 -seed weight showed significant positive better parent heterosis.

\section{Heterosis estimation}

The data for seed yield per plant revealed that nine and five crosses manifested significant positive relative heterosis and heterobeltiosis, respectively. The crosses that exhibited highly significant positive heterobeltiosis for seed yield were Pusa karishma x varuna (21.57**), PM-22 x RSPR-01 (20.00**), PM-22 x RSPR-03 (18.26**), PM-22 x RSPR-03 (7.32**) and Pusa karishma $x$ RSPR-01 (4.09**). Moderate level of heterosis for seed yield/plant, was also reported by Aher et al., (2009).

In the present study, negative mid-parent and better heterotic values for plant height were noted for three and eight crosses, respectively. Pourdad and Sachan (2003) also reported significant negative heterosis for plant height in Brassica napus. Similarly, Nassimi et al., (2006) also obtained significant negative better-parent heterosis for plant height.

In Brassica, positive heterosis for number of primary branches is desirable, because plants with vigorous stature containing more branches provide opportunity for higher yields. Significant positive heterosis for number of primary branches was earlier reported by Turi et al., (2006) and Nasrin et al., (2011). Similarly, for number of siliqua on main raceme, the significant positive better parent heterosis was observed for two crosses viz., Pusa karishma $x$ kranti and Nov gold $x$ Kranti with the values from 9.57 and 6.74 (\%), respectively. Eight crosses for siliqua length, four crosses for number of seeds per siliqua, and one cross for 1000-seed weight showed significant positive better parent heterosis.

From the foregoing discussion, it has become evident that majority of heterotic crosses for yield traits involved either one or both the good or average or poor GCA parents (Table 5). As such, several crosses are of specific significance, as besides being highly heterotic, they also gave good per se performance and involved parents with good GCA. Therefore, they can be exploited further for yield improvement. Such crosses are likely to throw transgressive segregants in advance generations.

\section{References}

Aher, C. D., Shelke, L. T., Chinchane, V. N., Borgaonkar, S. B. and Gaikwad, A. R. 2009. Heterosis for yield and yield components in Indian mustard [Brassica juncea (L.) Czern and Coss]. International Journal of Plant Science, 4(1): 30-32.

Dar, Z. A., Wani, S. A., Habib, G. M., Ali, G., Sofi, P. A., Dar, S. A. and Iqbal, A. M. 2013. Analysis of combining ability in brown sarson (Brassica rapa L.) under temperate conditions. African Journal of Agricultural Research 8: 1603-1607.

Kempthorne, O. 1957. An introduction to genetic statistics. John Wiley and Sons, Inc. New York, USA pp 468-473.

Nasrin, K., Fateha, N., Shahidar, R. B., Shahnaz, S. and Islam, M. 2011. Heterosis and combining ability in Indian mustard [Brassica juncea L. 
(Czern and Coss)]. Bangladesh Research Publication Journal, 6(1): 6571.

Nassimi, A. W., Raziuddin, S. A., Naushad, A. 2006. Study on heterosis in agronomic characters of rapeseed (Brassica napus L.) using diallel. Journal of Agronomy 5: 505-508.

Panse, V.G. and Sukhatme, P.V. (1967). Statistical Methods for Agricultural Workers. $2^{\text {nd }} E d$. New Delhi, ICAR, pp. 381.

Patel, A., Prajapati, K. P., Patel, P. J., Shah, S. K. and Patel, P. S. 2015. Genetic studies of yield and quality traits in Indian mustard (Brassica juncea L.). Journal of Oilseed Brassica, 6(2): 289-295.

Pingali, P. L. 1997. The role of heterosis in meeting world cereal demand in the $21^{\text {st }}$ century. Book of abstracts. The genetics and exploitation of heterosis in crops; An International symposium. CIMMYT, D.F. Mexico., pp: 348-349.

Pourdad, S. S. and Sachan, J. N. 2003. Study on heterosis and inbreeding depression in agronomic and oil quality characters of rapeseed (Brassica napus L.). Seed and Plant 19: 29-33.

Shull, G. H. 1952. Begginings of the heterosis concept. In: Heterosis, J. W. Gowen (ed.), pp. 14-48. Lowa State University. Press, Ames, IA.

Turi, N. A., Raziuddin, S., Shah, S. and Ali, S. 2006. Estimation of Heterosis for some important traits in Indian mustard [Brassica juncea L. (Czern and Coss)]. Journal of Agricultural and Biological Science, 1(4).

Turner, J. H. 1953. A study of heterosis in upland cotton, combining ability and inbreeding effects. Agronomy Journal, 43: 478-490.

Yadava, D. K., Singh N, Sujata V, Singh R., Singh S., Giri, S. C., Dwivedi, S. K. and Prabhu, K. V. 2012. Combining ability and heterobeltiosis for yield and yield attributing traits in Indian mustard (Brassica juncea). Indian Journal of Agricultural Sciences 82: 563-570.

\section{How to cite this article:}

Rai, S.K., Rubby Sandhu, Lekhraj Jat, Ashwani Kumar, Usha Kiran, Shazia Mukhtar, Surbhi Kohli and Rai, G.K. 2017. Study of Heterosis and Combining Ability for Yield and its Component Traits in Brassica juncea L. Int.J.Curr.Microbiol.App.Sci. 6(12): 2570-2579. doi: https://doi.org/10.20546/ijcmas.2017.612.298 